\title{
Collaborative Learning at Engineering Universities: Benefits and Challenges
}

\author{
https://doi.org/10.3991/ijet.v13i01.7811 \\ Olga V. Sumtsova $\left.{ }^{\bowtie}\right)$, Tatiana Yu.Aikina, Liudmila M. Bolsunovskaya \\ National Research Tomsk Polytechnic University, Tomsk, Russia \\ olgasumtsova0205@mail.ru \\ Chris Phillips \\ Newcastle University, Newcastle upon Tyne, United Kingdom \\ Olga M. Zubkova, Peter J. Mitchell \\ National Research Tomsk State University, Tomsk, Russia
}

\begin{abstract}
This paper concerns the cutting edge educational approaches incorporated into syllabuses of the most progressive Russian higher technical schools. The authors discuss one of the active methods in teaching foreign languages - collaborative learning implemented in e-courses. Theoretical and historical aspects of this approach are addressed, as are its suitability for engineering education and possible ways of introducing collaborative learning into ecourses. Collaborative learning technology offers wide prospects for teachers of foreign languages as it enables the use of various patterns of interaction, promotes discussion, opinion exchange, peer assessment and building an e-learning community, fosters the development of e-culture and netiquette, and prepares future specialists for work in their professional sphere under the new conditions imposed by society's technological and cultural development. This paper describes real pedagogical experience of teaching English to students using the platform Moodle, focusing on the capacity of different Moodle instruments for designing group work tasks. Recommendations are given for their usage and the results of implementing a collaborative learning approach into certain e-courses offered at Tomsk Polytechnic University (TPU) are presented.
\end{abstract}

Keywords - collaborative learning, e-learning, engineering education, Moodle

\section{Introduction}

The current state of socio-economic development in the Russian Federation determines the significant role of engineering education in solving the problems of modernization and integration of the country into the universal community. It is generally recognized that scientific and technical ideas, developments, high technology and science-intensive products as well as the intellectual and educational potential of human resources represent the mainspring of the sustainable economic and political development of the country. The formation of a global ground-breaking society is 
based on massive investment into human resources, progression of professional skills and scientific research along with the modernization of higher engineering education. Having this in mind, the overriding priority for the administration and faculty of any modern engineering university is to create appropriate conditions for training highly qualified professional engineers capable of competing internationally. It is necessary to note that this mission is utterly impossible without integrating up-to-date educational technologies into the learning process at Russian engineering universities.

E-learning emerged not so long ago and due to its novelty incorporates the best methodological practices and modern educational technology. Such popular educational technologies as team working, collaborative learning, dynamic cognitive process and working with various sources of information are currently viewed as the most advantageous and appropriate methods for e-learning. The aforementioned technologies are active methods of training and employ a wide range of research and problem-solving techniques, application of obtained knowledge in cooperative and individual activities, development of independent critical reasoning, communication standards and the ability to perform diverse social roles in collaborative activities.

The modern pedagogical community regards collaborative and cooperative learning as a key element in the process of successful knowledge and practical experience acquisition. Collaborative learning technology originated as an alternate form to the traditional teacher-centered or lecture-centered educational system. This technology brings together three main concepts in a single process: learning in teams, peer assessment and working in small groups.

The term "collaborative learning" refers to students' mutual (distributed) learning which encourages them to work together collectively designing or "producing" new scholastic attainments, rather than absorbing the knowledge provided by the instructor. This model implies the use of small groups of students in a real or virtual class. In this context class assignments are set in such a way that all members of the team become interdependent, but sufficiently autonomous to master the learning material as well as to solve specific problems.

The underlying idea behind collaborative learning is that knowledge is created by human communities and that learning occurs in the process of communication. Students process the obtained information into knowledge by swapping ideas, thoughts and feelings. As a result they come to realize what is acceptable and significant for other members of the community. Therefore it may be concluded that if a student learns to listen to and hear his/her peers, he/she will learn to construct his/her own knowledge.

\section{$2 \quad$ Literature review}

The main ideas of behind collaborative learning were developed by a group of teachers (Edwin Mason, Charity James, Leslie Smith) from Goldsmith's College, University of London in Great Britain in the mid-1960s. Later, in the 1980s, many progressive teachers all over the world took an interest and several teams of researchers from the University of Johns Hopkins, Minnesota State University and Jonathan 
David Aronson's team from California developed a detailed methodology for collaborative learning.

Since that time the problem of organizing the educational process by means of collaborative learning was studied and analyzed in the literature by scholars internationally to a great extent. Generally speaking, collaborative learning itself is hardly a new idea. The Russian psychologist Vygotsky (1978) was foremost amongst the pioneers who explored the causal relationships that exist between social interaction and individual learning [1].Later, collaborative learning was researched by the Russian educator E. S. Polat, who claimed that the main concept of collaborative learning referred to creating conditions for the active cooperative learning activity of students in various case studies. Experience has proven that collaborative learning makes the overall learning process more comprehensive, but much more challenging and effective. Moreover, effectiveness concerns not only the academic progress of the learners but also their science-driven growth along with ethical growth [2].

According to Zhu [3], collaborative learning is a social interaction that involves a community of learners and teachers where members acquire and share experience or knowledge. In collaborative learning, the central elements are positive interdependence, individual accountability, face-to-face and verbal interaction, social skills, and team reflection. As a result, students develop individual responsibility for group results [4]. The elements listed above are of particular interest within the framework of teaching engineering students since the nature of work in organizations is changing from individual assignments to team-based activities, and group work is becoming common in a variety of professions, especially in disciplines oriented towards design and development. In addition, as professional implementation is becoming more and more geographically distributed, online collaboration among team members has become regular practice [5].As for traditional language teaching, it involves teachercentered learning in which many of the ingredients of grammar-translation and audio lingual methods are employed. Such teaching concentrates on making students aware of certain aspects of language without providing sufficient practice. Collaborative learning, in contrast, shares some characteristics in common with communicative language teaching, which highlights both interaction and communication among students and between students and teachers[6].At the same time quite a limited body of research has been focused on how computer-supported collaborative learning can influence students' written communication. For these reasons, this study aims at understanding how collaborative learning can be used effectively in the context of Russian higher education.

\section{Computer-supported collaborative learning as a an effective tool in training modern engineers}

In recent years, collaborative learning has received a new interpretation within the context of e-learning - computer-supported collaborative learning. It incorporates several concepts: 
- learning becomes more productive when there is good social rapport between the members of the group;

- the ability to interact with other members of the group has an impact on the learners' ability to express their ideas properly, clearly and in a logical way;

- in the course of social interaction learners create an educational community of people possessing certain knowledge. This kind of mutual cognitive activity in the community serves as a basis of the synergetic effect of the learning method under study.

Collaborative learning is built on such learning theories as social constructivism and connectivism and is implemented through collaborative and cooperative methods of learning. These methods have a number of common features along with specific ones. Cooperative learning involves cooperation of learners in groups. Cooperative learning is a structured, systematic learning strategy where small groups of 3-5 purposefully picked learners work together on a collective goal creating a certain end product.

The peculiarity of cooperative learning is a system of carefully planned stepsin performing a particular task as well as certain rules of appropriate behavior at each step. Each student is in charge of the results of his/her own work whereas the teacher takes on the role of an adviser who is responsible for the whole process of cooperative learning.

As for collaborative leaning, it refers to a collaborative activity of two or more learners who are working on achieving collective goals through the exchange of knowledge, learning and reaching a consensus. Broadly speaking, collaborative learning applies to learning activities which include an exchange of views, discussion, mutual commenting and revision.

Collaborative learning is more goal-oriented in comparison with the cooperative system of organizing a learning activity and more centered on the teacher. Consequently, it requires more effort to plan.

Cooperative and collaborative learning organized in an e-learning environment suggest using interaction services (Web 2.0: Wiki, blogs, social networks, collaborative apps, virtual classes, Communities of Practice - CoP etc.) with the purpose of learning. A number of methods intended to promote unsupervised activities of learners in small groups have streamed from the ideas of constructivism and connectivism.

Working in small groups allows participants of the e-course to raise their cognitive interest and positive attitude toward the whole learning process. Students acquire knowledge on business communication; learn how to use the extensive opportunities of the Internet and traditional communication technology in order to obtain knowledge and solve a problem.

\subsection{The main way of implementing collaborative learning}

Among the main ways, methods and technologies of collaborative learning we can find team teaching, team research work, brainstorming sessions, role playing games, 
problem-based learning, project-based learning, debates, the Jigsaw method, case study, etc.

Collaborative learning also provides us with numerous patterns of interaction learners can use to acquire new knowledge. The most widely used are:

- debates in the process of discussing an issue or problem with their peers;

- teaching another student, when a student gains knowledge, presenting new material to his/her group mate(s);

- being taught by another student, when a student gains knowledge when taught by his/her group mate(s);

- observation, when knowledge acquisition results from observing peers undergoing the process of mastering new material;

- self-expression, when new knowledge is acquired in the process of presenting and explaining new material to other learners;

- reflection and recommendations, when new materials are covered in the course of peer reflection or error correction.

In that regard it is necessary to consider some benefits of collaborative learning:

1. students learn to work in teams and come to a consensus in spite of differing views, cultures or personality traits;

2. students are provided with the opportunity to appreciate various viewpoints and have a critical look at their own understanding of the problem;

3. students have the opportunity to solve problems and perform learning tasks in the online mode.

4. The possible challenges of the method under consideration may be the following:

5. a possible decrease in the team experience in case any member of the team responds slowly or is not engaged in teamwork at all;

6. dependence of individual team participant assessment on the assessment of the performance of the whole team;

7. collaborative types of activities can be considered ineffective if students fail to communicate and interact with their peers.

Some of the challenges listed above can be overcome by using different strategies of preparing students for collaborative activity before they get engaged in the learning process in earnest.

\subsection{Preparation for collaborative learning}

When learners undergo intramural studying in teams, their socialization occurs naturally. Students easily develop professional and social relationships with their peers and have no difficulty engaging in collaborative activities. However if students are far from each other (in case of distance learning), the teacher should use special strategies to help students form a learning community in the e-learning environment.

The easiest way to prepare students for collaborative learning is to schedule a faceto face meeting in the form of an inception meeting, introductory lecture or discussion 
at the very beginning of the learning process. If it is impossible to schedule a face-to face meeting, learners are invited to create a personal account on the corporate eresource (a group site or a blog) or answer questions reflecting their hobbies and interests, attitude to the urgent social and ethical problems. Questions at least partially related to the content of the e-course are highly recommended. Such learning events are beneficial not only for organizing students' interaction, but they also can help the teacher get to know the future participants of the e-course and plan their teamwork more thoroughly.

\subsection{Guidelines for developing tasks for teamwork}

In order to make teamwork effective and prepare appropriate tasks and assignments, teachers should follow several useful recommendations. To begin with, tasks for teamwork should be relevant to the course objectives. Students should realize that team activities are an integral part of the learning process and a means of achieving certain goals, but not just another way of keeping them engaged. Secondly, carefully planned teamwork should include several stages with an opportunity to consider or evaluate the results of every interim stage, if applicable. In this regard, it is recommended to set clear time limits for each stage in order to promote collaboration and facilitate all members of the team to take part. Also, it is not recommended to plan more than two team tasks per academic term, as it requires great effort on the part of both students and the teacher. Teamwork fits well in preparation for project activities, conference weeks and so on. Naturally, the assignments composed and uploaded in the e-course must correlate with the students' abilities and skills. The assignments can be made more complex as soon as the students have mastered new material and acquired relevant experience. Another important point is that the design of a team task should allow students to distribute the work in a team fairly. The tasks should be structured in such a manner that all members of the team have a chance to make an equal contribution to the team result. And finally, when planning the teamwork assessment procedure, the teacher should think how to assess each student's performance as well. For this purpose it is necessary to define the structure of the tasks clearly and foresee the possible learners' roles in the team.

\subsection{Elements of LMS Moodle for communication and collaborative activity}

Generally speaking, all the active elements of an LMS Moodle can be used for implementing methods of collaborative learning. Let us consider the most appropriate (according to our experience) elements of LMS Moodle and their potential for organizing collaborative activities.

The "Forum" instrument refers to the element enabling communication between the teacher and the students. It is designed to introduce various topics for discussion in asynchronous conditions. Working in "Forum" allows the participants of the ecourse to verbalize their ideas more accurately and analyze messages of other participants more thoughtfully. The mailing list of new messages helps the teacher to monitor all stages of the discussion. The "Forum" element enables the observation of the 
overriding principle of collaborative learning - working in a convenient place and at a convenient time.

Let us examine some forms of interaction based on the "Forum" element which could be practiced for implementing collaborative learning at engineering universities:

- A brainstorming activity organized in the "Forum" may contain two stages. The teacher sets the task in the description of the "Forum" and provides clear instructions. At the first stage learners have to generate ideas concerning the solution of the assigned task. Open discussion is prohibited at this stage. Then the teacher deletes recurring messages and allocates each idea to a fresh topic using the separation function. At the second stage students are encouraged to discuss the suggested topics and choose the ultimate solution of the problem.

- Debates are organized by the teacher who offers a number of problematic topics allowing ambiguous responses. At the beginning the teacher introduces rules for holding a discussion and assessment criteria for the students' replies. Then the students are divided into pairs and take on the roles of the opponents. The objective of each learner is to justify his/her own opinion within the scope of the topic under discussion. Upon completing the debates, the teacher assesses each student's performance according to the relevant criteria presented before.

Now we shall present a real assessment activity organized by means of the "Forum" element in LMS Moodle. All the learning activities presented in the paper were organized and conducted by the ESL teachers at TPU in groups of second-year students studying for their Bachelor's Degree in Mechanical Engineering.

\subsection{Sample 1.A panel discussion organized in the "Forum"}

A panel discussion usually includes three development stages: orientation, assessment, consolidation. The first stage is concerned with the process of "orientation" in the stated problem and adaptation to each other and the general atmosphere of the discussion on the part of the students taking part in the discussion. It is the stage of developing a certain approach to solving the stated problem. The "assessment" stage resembles the situation of considering and comparing information and various positions, and generating ideas. The last stage - "consolidation" -is intended to work out unified or compromise solutions, views and positions. Thus a "Forum"-based panel discussion may include the following steps:

1. a thematic forum is created where the problem for discussion is posted;

2. the teacher divides the students into micro groups (6-8 students);

3. each micro group chooses its representative;

4. each micro group creates its discussion theme within the suggested "Forum" problem. As a result of the discussion members of each group come to a unified standing on the problem;

5. the teacher creates a new theme in the "Forum" intended for summarizing the results of group discussions where representatives of each group discuss their 
group's positions on the stated topic in accordance with the given criteria (e.g. strengths and weaknesses, possibility for further implementation, feasibility of the suggested solutions) whereas other learners simply follow the course of the discussion;

6. the teacher summarizes the results of the discussion presenting all the positions and the common solution if applicable.

The "Seminar" instrument presents a learning activity where each student not only performs his/her own work but also evaluates the results of the work performed by other learners. The "Seminar" includes several phases, each of which has its own tuning parameters and materials. The list of phases of a seminar is provided below. Each phase is activated by the teacher.

1. Settings of the seminar.

2. Allow the students to upload their work.

3. Allow the students to upload their work and assess.

4. Allow the students to assess the work of their peers.

5. Provide the students with a final assessment.

Final assessment in the "Seminar" instrument takes into account not only the quality of the students' own work, but the students' activity as reviewers as well. The «Seminar" is one of the most complicated instruments of LMS Moodle and includes a great variety of settings which allow the determination of an assessment strategy as well as criteria for assessment and self-assessment of the students' activity as learners and reviewers.

\subsection{Sample 2. A peer assessment learning activity organized in the "Seminar"}

Here we present a learning activity organized using the "Seminar" instrument and implemented in the e-course for second-year students studying for their Bachelor's Degree in Mechanical Engineering at TPU.

\section{Description of the parameters}

- Discipline: Foreign language (English)

- Target audience: Second-year students of the Institute of High Technology Physics

- Level of English proficiency: B1

- Writing: an argumentative essay (Engineering is my future profession: pros and cons/The pros and cons of Engineering as a future profession)

- Learning Outcomes: practice and consolidation of the active vocabulary on the topic "Introduction into the professional activity: my future profession", development and improvement of writing skills (an argumentative essay expressing a personal opinion).

- Workload: 8 academic hours (4 hours for working on the essay, 2 hours for peer assessment, 2 hours for working in the "Forum": assessment comments, feedback).

- Form implementation: Electronic environment. 
Table 1. Description of the learning activity, instruments, services

\begin{tabular}{|c|c|c|}
\hline Description of the learning activity & \begin{tabular}{|c} 
Desired services for organizing a \\
learning activity(learning materials \\
/ software)
\end{tabular} & \begin{tabular}{|c} 
LMS Moodle \\
instruments and \\
Web 2.0 services, \\
appropriate for \\
implementation of \\
the given activity \\
in the electronic \\
environment \\
\end{tabular} \\
\hline $\begin{array}{l}1 \text { Read and analyze the study guide "Planning } \\
\text { and writing essays" (page 11) as well as rec- } \\
\text { ommendations for essay writing provided in the } \\
\text { e-course "The English language. Module 3" }\end{array}$ & $\begin{array}{l}\text { The study guide for writing essays } \\
\text { "Planning and writing essays", the e- } \\
\text { course "The English language. } \\
\text { Module 3", recommendations for } \\
\text { essay writing } \\
\text { http://design.lms.tpu.ru/mod/assign/v } \\
\text { iew.php?id=123402 }\end{array}$ & $\begin{array}{l}\text { Moodle Instru- } \\
\text { ments "Seminar" } \\
\text { and "Forum" }\end{array}$ \\
\hline $\begin{array}{l}2 \text { Follow the useful links provided and look } \\
\text { through the information concerning the topic } \\
\text { under study. }\end{array}$ & $\begin{array}{l}\text { Useful links for facilitating the work } \\
\text { on the essay (free excess): } \\
\text { http://www.engineeringedu.com/Cele } \\
\text { stesBlog/blog/2013/01/14/pros-and- } \\
\text { cons-of-an-engineering-degree/ } \\
\text { http://learningpath.org/articles/Engin } \\
\text { eer_Career_Overview.html } \\
\text { https://www.quora.com/What-are- } \\
\text { the-pros-and-cons-of-being-an- } \\
\text { engineer } \\
\text { https://answers.yahoo.com/question/i } \\
\text { ndex?qid=20070328192906AAO60 } \\
\text { Ni }\end{array}$ & \\
\hline $\begin{array}{l}3 \text { Write an essay ( } 200-250 \text { words) on the fol- } \\
\text { lowing topic: Engineering is my future profes- } \\
\text { sion: pros and cons. }\end{array}$ & & \\
\hline $\begin{array}{l}4 \text { Upload your works into the "Seminar" (docx. } \\
\text { format). }\end{array}$ & & \\
\hline $\begin{array}{l}5 \text { Read and understand the assessment criteria } \\
\text { related to writing the essay provided in the e- } \\
\text { course "The English language. Module 3". }\end{array}$ & $\begin{array}{l}\text { Assessment criteria, the e-course } \\
\text { "The English language. Module } \\
\text { 3".http://design.lms.tpu.ru/mod/assig } \\
\text { n/view.php?id=123402 } \\
\end{array}$ & \\
\hline $\begin{array}{l}6 \text { Choose an essay written by one of your } \\
\text { groupmates, review and assess it in accordance } \\
\text { with the criteria. }\end{array}$ & & \\
\hline $\begin{array}{l}7 \text { Upload your assessment table (docx. format) } \\
\text { into the "Seminar". }\end{array}$ & & \\
\hline 8 Comment on your marks in the "Forum". & & \\
\hline $\begin{array}{l}9 \text { Feedback: post your responses to your } \\
\text { groupmates' marks in the "Forum". }\end{array}$ & & \\
\hline
\end{tabular}

\section{Instructions for learners' interaction in the electronic environment:}

1. Choose an essay of one of your peers and assess it according to the criteria provided by the teacher. 
2. Comment on your assessment in the "Forum".

3. Read the comments of other learners and respond.

Having conducted the learning activity described in the table above, we would like to share the results of our work. Forty-eight students (an experimental group) who took part in the activity were asked to fill in a special questionnaire afterwards to reveal some of the challenges they faced working in the "Seminar". The questionnaire included questions concerning the work at each stage and the complexity degree of each task.

Questionnaire (original version for the students). The main purpose of this questionnaire is to reveal possible challenges you faced working in the "Seminar" in order to improve the activity and avoid them in the future. Please evaluate the degree of complexity of each stage of the learning activity you have just completed. Degree of complexity (from 1 to 5): 1 - very easy, 2 - easy enough, 3 - quite difficult, 4 very difficult, 5 - impossible to complete. In the Feedback column explain the reasons behind challenges you faced.

Table 2. Questionnaire

\begin{tabular}{|c|c|c|}
\hline Stage of the task & $\begin{array}{c}\text { Complexity degree } \\
(1-5)\end{array}$ & Feedback \\
\hline \multicolumn{3}{|l|}{$\begin{array}{l}\text { Reading and analyzing the study guide "Planning and writing } \\
\text { essays" as well as recommendations for essay writing provided } \\
\text { in the e-course "The English language. Module 3" }\end{array}$} \\
\hline \multicolumn{3}{|l|}{$\begin{array}{l}\text { Studying the information concerning the topic under study } \\
\text { (online) }\end{array}$} \\
\hline \multicolumn{3}{|l|}{$\begin{array}{l}\text { Writing an essay (200-250 words) on the following topic: Engi- } \\
\text { neering is my future profession: pros and cons. }\end{array}$} \\
\hline \multicolumn{3}{|l|}{ Uploading the essays into the "Seminar" (docx. format). } \\
\hline \multicolumn{3}{|l|}{$\begin{array}{l}\text { Reading and understanding the assessment criteria related to } \\
\text { writing an essay provided in the e-course "The English language. } \\
\text { Module 3". }\end{array}$} \\
\hline \multicolumn{3}{|l|}{$\begin{array}{l}\text { Choosing an essay written by one of your groupmates, reviewing } \\
\text { and assessing it in accordance with the criteria. }\end{array}$} \\
\hline \multicolumn{3}{|l|}{$\begin{array}{l}\text { Uploading the assessment table (docx. format) into the "Semi- } \\
\text { nar". }\end{array}$} \\
\hline \multicolumn{3}{|l|}{ Commenting your marks in the "Forum". } \\
\hline $\begin{array}{l}\text { Feedback: posting your responses to your groupmates' marks in } \\
\text { the "Forum". }\end{array}$ & & \\
\hline
\end{tabular}

\section{Questionnaire outcomes:}

1. The theoretical part of the leaning activity under review connected with studying information in the textbook and running through recommended Web sites was very easy for forty students (83\%). But eight learners (17\%) found the theoretical part very difficult, boring and time-consuming.

2. The practical part of the activity (essay writing) was considered easy enough and satisfying by thirty-nine students $(81 \%)$ whereas nine learners $(9 \%)$ considered es- 
say writing very difficult to accomplish, feeling their lack of appropriate writing skills.

3. The peer assessment part of the activity turned out to be the most complicated assignment: only twenty-five students $(52 \%)$ managed to complete it successfully with permissible variation from the criteria. Twenty respondents $(42 \%)$ demonstrated not high but quite satisfactory results. Meanwhile three students $(6 \%)$ failed this part completely being unable to assess their peers' essays.

Summarizing the task, thirty-two respondents $(67 \%)$ claimed that they were absolutely satisfied with their own performance in the activity as well as the results of peer assessment. They described the stage of writing the essay as the easiest one and the stage of writing reviews as the most challenging but rewarding at the same time. On the other hand, sixteen students $(23 \%)$ faced difficulties in writing reviews and assessing the essays of their peers as they had not had much experience of working in such a mode before. As a solution of the problem, the teacher was advised to upload some review samples into the "Seminar" as well as organize a special consultation "Forum" at the stage of reviewing and assessing, where learners would be able to ask questions arising during the work.

III. The "Wiki" instrument

Wiki is a useful instrument suitable for organizing teamwork and collaborative learning in general. Wiki represents a set of webpages, one of which is the front page and, as a rule, moving to all the other webpages is carried out from it. Each Wiki webpage has its own forum for posting comments and a change log which provides an opportunity to monitor students' activity. Wiki might be a cooperative resource (any participant of the e-course has the right to add webpages, edit them and post comments) or a private resource allowing only a particular group of students to create the content.

\subsection{Sample 3. Problem-based learning organized with the help of the "Wiki" instrument}

1. The teacher posts a description of the problem-based situation on the "Task" webpage, procedure of working on the solution of the problem and assessment criteria.

2. The students have to study the problem-based situation on their own at home and write a report including various ways of solving the problem.

3. Then the teacher divides the group into several sub-groups of 4-5 students. He/she creates an individual Wiki resource for each team where the students post their reports.

4. The students discuss the situation in their sub-groups, posting comments.

5. The representatives of each sub-group assess and summarize the considered versions, choosing the best one for presentation to the teacher in the "Forum".

6 . The teacher reviews the results and makes conclusions. 


\subsection{Support technology for collaborative learning: asynchronous and synchronous interaction in the Moodle environment}

In contrast to face-to-face activities, teamwork organized in the e-learning environment is associated with interaction which can be described as "human - computer - human" and may be carried out in two modes: synchronous and asynchronous. The choice of one of them or both modes simultaneously determines the level of learners' interaction and technology involvement into the learning process.

The level of interaction can be low, medium or high. When the interaction level is low, learners do not come into direct contact with other participants of the e-course; rather they use the results of their activity as a point of reference. When the interaction level is medium learners communicate by means of completing asynchronous types of tasks, and the results of their activity become an impulse that stimulates interaction between all the other participants of the team. When the interaction level is high, communication between the learners occurs in the synchronous mode, in other words, an immediate response is expected.

Let us consider how different types of tasks correlate with the Moodle instruments supporting various levels of interaction studied above.

Low level of interaction, asynchronous task types:

- The "Query" instrument allows the holding of a quick vote and queries among the participants of the e-course and presents responses in a percentage rating or diagrammatically. The results of the query can be used as an impulse for a classroom discussion as well as a general conclusion related to the results of teamwork.

- A "Glossary" is used to create a list of words, terms, quotations and so on. It links words and expressions throughout the whole content of an e-course with their meanings in the "Glossary". Working on the "Glossary" occurs individually, but the results are available for all the participants.

- Medium level of interaction, asynchronous task types:

- The "Wiki" instrument can be used for developing joint products/projects. It provides all the participants with the opportunity to take part in editing the project. The product development is based on new information added by the participants to that already uploaded.

- The "Forum" instrument is intended for a synchronous information exchange among all the participants of the learning process. It can be used for a topic thread.

- The "Seminar" instrument is designed for organizing mutual assessment among the participants.

- High level of interaction, synchronous task types:

- The "Chat" instrument is used for sharing information among all the participants of the learning process and online discussions in the written form.

- The "Videoconference/webinar" Instrument allows the introduction of such learning modes as seminars, lectures and presentations into the e-course by means of live stream and promotes oral communication between the participants. 


\subsection{Aspects of organizing collaborative activities}

Let us consider some aspects of the teacher's work when organizing students' collaborative learning activities in an e-environment.

1. The content aspect is focused on solving group problems - sharing information, collaboration for product development as well as determining activity areas and working out ways of implementation.

One of the advantages of using Moodle in realizing the content aspect of group work is a wide range of communication tools- the platform maintains exchanging files of any format, it enables quick information supply to all the participants of the course or particular groups about current events and helps to organize a discussion of a stated problem, which can be conducted within separate groups. The platform may also serve as a basis for employing various types of projects: research, investigation, communication-oriented.

The most efficient model for implementing collaborative learning in the framework of full-time education is the integrated form: a combination of classroom-based and network interaction stages within group work (which corresponds to web-supported learning or blended learning).

When collaborative activities are organized in the integrated learning form, it is important to plan the distribution of the learning material between classroom work and collaborative activities in the electronic environment; to determine roles and functions of the learners and the teacher in the course of collaborative activities implementation; to sequence assignments and statements; to develop step-by-step instructions, including those related to performing activities on the e-platform basis.

The following elements of the content aspect may affect the efficiency of collaborative learning:

- material selection and structuring (incorporating the assignment system);

- selecting an appropriate platform instrument for working on various types of assignments;

- formulating assignments, requirements and instructions;

- the course structure logic and uniformity in particular units of the course.

2. The qualitative aspect presents the collaborative efforts of the group members (both the teacher and the students) in forming a learning community: changes in the participants' way of thinking, development of their ability to perform various roles and functions, elaborating their collaboration skills and fostering a group culture in the electronic learning environment.

The learners must become aware that their behavior and communication in the electronic environment, just like during interaction in the classroom setting, should follow certain rules, or so-called netiquette (from the English "net" and "etiquette"), or network etiquette. The major purpose of netiquette (observance) is to promote easier communication within a virtual community. Rules can be formulated according to the group's objectives, technological capacity, etc. In cases of integrated learning, it is preferable to work out the rules of communication on the platform in class. These rules should refer both to psychological/emotional aspects of communication, and 
technical ones (how to document the results of collaborative work, how to upload/download files).

3. The structure aspect aims at determining the entry list, collaborative work duration, roles and position structures within the groups.

There are three group modes on the Moodle platform that are suitable for organizing group work:

- "No groups" - the students are not divided into groups, everyone is part of one big community;

- "Separate groups" - the students of each group assume that their group is the only one, they cannot observe the performance of other groups;

- "Visible groups" - the students act within their group, but they can also see what is happening in other groups [7].

These technological capacities make it possible to organize various forms of group interaction: group wide, pair work, and work in small groups.

The platform allows the carrying out of both short-term and long-term projects. Each student's roles and functions are determined by the objectives of a particular assignment.

4. The coordination aspect is the most important supportive aspect of group work in the electronic environment. It involves planning, organizing and controlling group work in the electronic educational environment. The introduction of information and communication technology to the educational process has created a new role for the teacher, which can be characterized as e-learning maintenance.

The teacher is supposed to be able not only to design a logical course but also to assist and consult the students, organize their work and encourage their independent learning in the e-environment.

\subsection{Maintenance of collaborative work provided by the teacher}

Let us consider maintenance of collaborative work provided by the teacher on the example of developing an e-course for the teaching program that has recently been implemented at TPU "Designing academic activity based on blended learning".

Creating and maintaining a facilitative team environment within the learning group by means of cognitive and social processes management is one of the teacher's key functions, which enables the building of a learning community serving as a basis of "student - student" interaction in the electronic course.

A learning community is essential in forming a productive communication environment, sharing information and promoting learners' cooperation for the purpose of achieving higher learning efficiency and performance. A learning community enables the employment of active methods of collaborative learning putting into practice the principle of "learning by teaching others".

The social "presence" of the teacher is manifested in particular methods of supporting the learning community and is provided through:

- activities for creating a favorable micro-climate in the group; 
- selecting and applying appropriate communication services;

- devising assignments for peer assessment or discussion of the end products;

- designing and maintenance of group work activities.

The following steps are recommended for the teacher to create a special team environment in ELE:

- a lead-in forum for students to introduce themselves, to discuss the discipline agenda, expectations of learning and other issues. Short messages with compulsory peer commenting solve the problem of lack of face-to-face contact and enable the involvement of the majority of learners in the discussion at the early stage;

- input and output surveys among learners make it possible for the teacher to make a group profile, to elicit problem areas and initiate communication regarding group members' concerns;

- building up a polite atmosphere through discussing the rules and norms of netiquette.

When the teacher maintains and coordinates group work with the learners on the elearning platform, his/her role involves regularly monitoring in-group processes, each group member's activity, analysis and addressing the causes of failures in collaborative activity performance. Problems in performing collaborative work in the eenvironment can occur when the group participants work asynchronously or disregard assignment deadlines due to various external and internal reasons. To avoid the aforementioned situations, it is reasonable to provide clear instructions, come to agreement about actions in case of technical problems, and impossibility of completing a task because of illness or language difficulties. It is important to discuss these matters in class in advance.

One of the most important elements in organizing and maintaining collaborative work by the learners is a set of instructions. This is a set of requirements providing a step-by-step guideline for successful activity completion, assessment, as well as assessment criteria for peer evaluation and correction, samples and the best pieces of work. All the aforementioned elements designed once can be applied (with minor modification) over a long period of time.

When writing the instructions, it is recommended:

1. to formulate instructions briefly, but still with sufficient information capacity. This can be achieved by means of text multilayering. A text multilayer is created by adjusting hyperlinks between files and elements of LMS Moodle which makes the instructions short but gives opportunity for clickthrough to the requirements, criteria, recommendations, sample files and typical mistakes, etc.;

2. to provide a transparent instruction structure using a step-by-step study guide;

3. to achieve the effect of the teacher's virtual presence. For example, the statements that students read from the screen can be "humanified" with the help of the teacher’s emphasis remarks: "Important!", "Pay attention!", "Remember!" 


\subsection{Methods of peer assessment}

Finally, the key elements of organizing collaboration in ELE are activities aimed at peer evaluation: peer commenting, peer revision and peer assessment.

Peer commenting - the simplest form of peer cross-check - is an element of a free discussion on a creative, problem-solving activity, essay and others. As a rule, rigid requirements are not applicable to peer commenting. It means expressing one's own opinion in the free form of recommendations, suggestions, and reasoned arguments in continuation of the author's writing or opposing his/her position. Peer commenting is an element of a discussion assuming a compulsory respond from the author. To avoid formalization it is recommended to set parameters for commenting. The requirements may concern: length of the comment, asking a question of the author, compulsory recommendations, and a discussion of strong/weak points of the end-product. The assignment should set a direction, parameters or a mini-scenario of a comment, which will not allow the students to simply write "everything is fine". The LMS Moodle tool "Forum" as well as any social networking tools (social walls) are appropriate for organizing peer commenting.

Peer revision is a reasoned comment based on the criteria applicable to an essay. Consequently, it is necessary to formulate clear assessment criteria on which a review will be based. When setting the criteria, it is recommended to:

- avoid using straightforward closed question (yes/no); they make the review formal;

- encourage the reviewer to give comprehensive answers;

- recommend to generalize a conclusion about the written work, or ask a question of the author;

- form matrix - tables to make peer revision convenient.

Carefully formulated criteria for peer revision may help to turn it into an additional form of group work with the learning material.

Peer assessment differs from peer revision by giving points for meeting the specified requirements for written works with specified terms of scoring. Devising valid criteria for peer assessment is a complicated matter for the teacher; however carefully formulated criteria enable achieving the veracity of the peer assessment result, making it comparable with the result of assessment by the teacher.

\section{Conclusion}

The issue considered in the paper concerns one of the most advanced topics related to higher technical education in the Russian Federation to-date, as the modern global society requires young specialists of great erudition possessing not only professional skills but also being able to collaborate with overseas colleagues without any assistance. The authors presented their experience of employing collaborative learning at Tomsk Polytechnic University. Special attention was paid to the benefits and challenges of the approach and its applicability for e-based education. 
Without doubt collaborative learning is an active method of teaching and learning and is considered to be particularly effective as it combines all the recent trends and developments in the field of mastering not only foreign languages but also other important engineering disciplines as well as humanities subjects. Collaborative learning promotes the development of communication skills, individual reasoning, communication culture and the ability to perform various social roles in collaborative activities.

In an e-learning environment, collaborative learning is essential for establishing an e-learning community and the development of socio-cultural skills in a foreign language, as well as mastering the language itself. Organizing group work in an eenvironment poses many challenges to the teacher and the learners, but could be overcome by careful planning, setting clear instructions, and selection of appropriate content material and task types, suitable Moodle instruments and assessment structure.

\section{$5 \quad$ References}

[1] Roberts, T.S. (2004). Online Collaborative Learning: Theory and Practice. Central Queensland University, Australia, 256-261 https://doi.org/10.4018/978-1-59140-174-2

[2] Polat, E.S. About the Methodology of Collaborative Learning. http://fralla.nethouse.ru/ articles $/ 20150$

[3] Zhu C. (2012). Student Satisfaction, Performance, and Knowledge Construction in Online Collaborative Learning. Educational Technology \& Society, 15 (1), 127-136.

[4] Leite Maria C. (2009). The impact of cooperative learning in English language learners' academic achievements

[5] Chiong, R. \&Jovanovic, J. (2012). Collaborative Learning in Online Study Groups: An Evolutionary Game Theory Perspective. Journal of Information Technology Education: Research, 11, 81-101 https://doi.org/10.28945/1574

[6] Zhang, Y. (2010) Cooperative language learning and foreign language learning and teaching.

[7] Condon, S. (2012). Moodle for Collaborative Learning.http://www.slideserve.com/lona/ moodle-for-collaborative-learning

[8] Aparicio, M. Bacao, F. \& Oliveira, T. (2016).An e-Learning Theoretical Framework. Educational Technology \& Society, 19 (1), 292-307

[9] Brindley, J.,Walti, C. \&Blaschke, L. (2009).Creating Effective Collaborative Learning Groups in an Online Environment. International Review of Research in Open and Distance Learning, 10(3) https://doi.org/10.19173/irrodl.v10i3.675

[10] Ellis, R. A., Goodyear, P., Prosser, M. \& O'Hara, A. (2006). How and what university students learn through online and face-to-face discussion: Conceptions, intentions, and approaches. Journal of Computer Assisted Learning, 22

[11] Goyal, E., Tambe, S. (2015). Effectiveness of Moodle-enabled Blended Learning in Private Indian Business School Teaching Niche Programs The Online Journal of New Horizons in Education, 5, (2), 14-22

[12] Plekhanova, M.V., Pigareva, E.P. (2012). Features of Students' Group Work Organization on the Basis of Electronic Platform Moodle in Process of Foreign Language Teaching in Non-linguistic Higher Education Establishment Pedagogy. Issues of Theory and Practice. Tambov: Gramota, 5 (16), 144-149 


\section{Acknowledgment}

The research is carried out at Tomsk Polytechnic University within the framework of Tomsk Polytechnic University Competitiveness Enhancement Program grant.

\section{$7 \quad$ Authors}

Olga V. Sumtsova is a senior lecturer at the Department of Foreign Languages of the Institute of Physics and Technology, National Research Tomsk Polytechnic University (Lenin Ave, 30, 634050, Tomsk, Russia).

Tatiana Yu. Aikina is an associate professor at the Department of Foreign Languages of the Institute of Natural Resources, National Research Tomsk Polytechnic University (Lenin Ave, 30, 634050, Tomsk, Russia).

Liudmila M. Bolsunovskaya is an associate professor, a chairperson at the Department of Foreign Languages of the Institute of Natural Resources, National Research Tomsk Polytechnic University (Lenin Ave, 30, 634050, Tomsk, Russia).

Chris Phillips is a Professor and SAgE Director of Diversity at the School of Computing, Newcastle University (NE1 7RU, Newcastle upon Tyne, United Kingdom).

Olga M. Zubkova is a senior lecturer at the Department of Foreign languages, National Research Tomsk State University (Lenin Ave, 36, 634050, Tomsk, Russia).

Peter J. Mitchell is an associate professor at the Department of Foreign languages, National Research Tomsk State University (Lenin Ave, 36, 634050, Tomsk, Russia).

Article submitted 09 October 2017. Published as resubmitted by the authors 23 November 2017. 